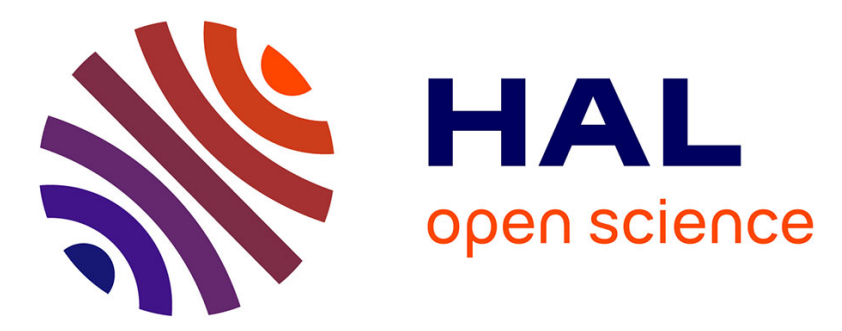

\title{
Driver's emotional state and detection of vulnerable road users: towards a better understanding of how emotions affect drivers' perception using cardiac and ocular metrics
}

\author{
Alex Lafont, Joceline Roge, Daniel Ndiaye, Jean-Michel Boucheix
}

\section{To cite this version:}

Alex Lafont, Joceline Roge, Daniel Ndiaye, Jean-Michel Boucheix. Driver's emotional state and detection of vulnerable road users: towards a better understanding of how emotions affect drivers' perception using cardiac and ocular metrics. Transportation Research Part F: Traffic Psychology and Behaviour, 2018, 55, pp.141-152. 10.1016/j.trf.2018.02.032 . hal-01768241

\author{
HAL Id: hal-01768241 \\ https://hal.science/hal-01768241
}

Submitted on 17 Apr 2018

HAL is a multi-disciplinary open access archive for the deposit and dissemination of scientific research documents, whether they are published or not. The documents may come from teaching and research institutions in France or abroad, or from public or private research centers.
L'archive ouverte pluridisciplinaire HAL, est destinée au dépôt et à la diffusion de documents scientifiques de niveau recherche, publiés ou non, émanant des établissements d'enseignement et de recherche français ou étrangers, des laboratoires publics ou privés. 
1 Lafont, A., Rogé, J., Ndiaye, D., \& Boucheix, J.-M. (2018). Driver's emotional state and

2 detection of vulnerable road users: Towards a better understanding of how emotions

3 affect drivers' perception using cardiac and ocular metrics. Transportation Research Part

4 F: Traffic Psychology and Behaviour, 55, 141-152.

$5 \quad$ https://doi.org/10.1016/j.trf.2018.02.032

6

$7 \quad$ Alex Lafont (corresponding author)

8 Univ Lyon, IFSTTAR, TS2, LESCOT, F-69675, LYON, France

$9 \quad$ Email: alex.lafont@ifsttar.fr

Phone : +33(0)4 72146909

Joceline Rogé

Univ Lyon, IFSTTAR, TS2, LESCOT, F-69675, LYON, France

Email: joceline.roge@ifsttar.fr

Phone : +33(0)4 72142419

\section{Daniel Ndiaye}

Université Paris-Est, COSYS, LEPSIS, IFSTTAR, F-77447 Marne-la-Vallée, France

Email: daniel.ndiaye@ifsttar.fr

Phone : +33(0)4 72146888

21

Jean-Michel Boucheix

LEAD-CNRS, University of Bourgogne Franche-Comté, F-21065-Dijon, France

Email: jean-michel.boucheix@u-bourgogne.fr 
Driver's emotional state and detection of vulnerable road users: towards a

better understanding of how emotions affect drivers' perception using

cardiac and ocular metrics

33

Traditionally, anger has been considered to have a detrimental effect on driving. However, recent studies suggest that this statement should be qualified, especially where vulnerable road user detection is concerned. One primary factor which may determine anger's effect on a driver's attention is its intensity. In the present study, different intensities of anger were elicited via film clips, then performances in vulnerable road user detection (i.e., of cyclists) were assessed while participants drove a car in a simulated environment. Cardiac and ocular measurements and self-reported data were used in order to accurately assess emotional state and attention management throughout the experiment. Results suggested that participants resorted to reappraisal strategies when they were exposed to the emotional film clips. This phenomenon did not directly affect cyclist detection performances, but evidence of different visual scanning strategies between groups emerged. The contribution of cardiac and ocular measurements to emotional assessment and the advantages of appraisal approaches of emotion were also discussed.

Keywords: anger; vulnerable road users; driving simulation; autonomic nervous system; heart rate variability; eye-tracking

\section{Introduction}

\subsection{Context}

Nowadays, many wheeled vehicles share the same space, and recent statistics have highlighted a human failure in managing the numerous interactions between road users. In 2015, the number of deaths on French roads increased for the second time in a row, by $1.7 \%$ compared to 2014. More specifically, pedestrians and cyclists, who are considered to be vulnerable road users (VRU), represented $18 \%$ of deaths in 2015, just behind motorists (52\%) and motorcyclists (22\%) (The French Road Safety Observatory, 2016). The same report also 
shows that the main causes of accidents involving pedestrians and cyclists are collisions with other road users, mostly motorists (69\% and 52\% respectively). In addition to the traditional factors involved in a crash (e.g., speed, inappropriate drinking), a lack of VRU visibility has also been reported (The French Road Safety Observatory, 2016). Consequently, the aim of the present research was to investigate the cognitive processes which affect the way drivers detect cyclists, whose presence on the streets of French cities is on the increase (Bouaoun, Haddak, \& Amoros, 2015). As attention management is required to detect some stimuli while carrying out a second task (Wickens, 2002), the exploration of the online processes which draw an individual's attention to a VRU during driving, especially when drivers are asked to detect VRUs, appears worthy of interest. In this context, conspicuity, which is the inherent ability of the stimuli to attract an individual's attention (Engel, 1971) emerges as an intriguing concept.

\subsection{Conspicuity and attention in driving}

"Two main types of attention are commonly distinguished in the literature: bottom-up or stimulus-driven and top-down or goal-oriented attention" (Nikolla, Edgar, Catherwood, \& Matthews, 2018). Bottom-up attention is determined by the physical characteristics of the information attended to, whereas top-down attention is guided by the observer's goals.

The earliest studies investigating conspicuity focused on the physical characteristics (e.g., shape, brightness, color, size) that make stimuli salient from their surroundings (Hancock, Wulf, Thom, \& Fassnacht, 1990; Wulf, Hancock, \& Rahimi, 1989). When this is the case, attention is mainly guided by bottom-up processes and is linked to the concept of sensory conspicuity.

More recent studies, notably related to research in the driving field, have shown that the focus of attention can also be influenced by expectations, objectives and knowledge relating 
to a stimulus (Hole, Tyrrell, \& Langham, 1996; Magazzu, Comelli, \& Marinoni, 2006; Rogé et al., 2017; Rogé, Douissembekov, \& Vienne, 2012). When this is the case, top-down processes associated with the concept of cognitive conspicuity, are involved in perception. According to Rogé, El Zufari, Vienne and Ndiaye, (2015), other components such as the driver's emotional state might also play a role in driving activity, especially VRU detection abilities. Therefore, the study of emotions could lead to a better understanding of situations in which motorists have to detect VRUs

\subsection{Emotion and attention in driving}

Previous studies have shown that emotion can affect a driver's attention management while driving. According to Ellis and Moore (2005), negative emotions are likely to elicit thoughts which are unrelated to driving activity. This phenomenon tends to decrease overall attentional resources. This in turn leads to inattention toward key activities (which are essential to ensure safe driving), for instance the detection of other road users (Regan, Hallett, \& Gordon, 2011). Several studies which are consistent with this idea, have demonstrated that negative emotions can impair driving. Pêcher, Lemercier and Cellier (2011), for example, showed that sadness was associated with degradation of performance due to irrelevant thoughts such as rumination and self-focus while driving. According to Jeon, Walker and Yim (2014), fear (assessed through Likert-type scales) can reduce drivers' attentional focus. Nesbit, Conger and Conger (2007) highlighted a link between anger and aggressive driving. Additionally, Garrity and Demick (2001), showed that anger, assessed using the NEO Personality Inventory (NEO$\mathrm{PI}-\mathrm{R})^{1}$ and the Profile of Mood States (POMS) ${ }^{2}$, led to an overall negative driving behavior. According to Jeon, Walker and Gable (2015), anger assessed via Likert-type scales could also

${ }^{1}$ (Costa \& McCrae, 1992)

2 (McNair et al., 1992) 
trigger dangerous modulations in driving style. Stephens, Trawley, Madigan and Groeger (2013) used an impeding vehicle and close rear traffic to elicit anger in participants who had to drive in a simulator in order to specifically study road user detection abilities. Using the Profile of Mood States Short Bilingual Version (POMS SBV) ${ }^{3}$ to assess emotions, they observed that anger-provoked drivers spent a considerably smaller proportion of time initially looking at less apparent pedestrians or emerging vehicle events than control drivers. They also took longer to make corrective actions to avoid potential collisions. Anger-provoked drivers tend to resort to a more superficial processing of potential hazards, and consequently underestimate the inherent risk of certain driving situations.

Contrary to these studies which underscore the detrimental effects of negative emotions - especially anger - during driving, other studies highlight the positive effects of anger on driving activity. Techer, Jallais, Fort and Corson (2015) used the Attention Network TestInteractions (ANT-I) paradigm ${ }^{4}$ which is a single task allowing the assessment of different attentional networks at the same time. They used ANT-I after eliciting anger through autobiographical recall procedure and checking the efficiency of their emotional induction via Affect Grid $^{5}$ and Brief Mood Introspection Scale (BMIS) ${ }^{6}$. They found a positive impact of anger on the alerting network which is dedicated to the preparation and sustainment of alertness when a high priority signal needs to be processed. According to Posner and Petersen (1990) this network is one of three independent networks of attention (i.e., alerting, orienting, and executive control). The authors concluded that anger could broaden the scope of the attentional focus via the alerting network.

\footnotetext{
${ }^{3}$ (Cheung \& Lam, 2005)

${ }^{4}$ (see Callejas, Lupianez, Funes, \& Tudela, 2005)

${ }^{5}$ (Russell, Weiss, \& Mendelsohn, 1989)

${ }^{6}$ (Mayer \& Gaschke, 1988)
} 
Rogé et al. (2015) presented safety messages in order to improve the perception of VRU vulnerability during driving. They assessed emotional state using an adapted version of the Geneva Emotional Wheel (GEM) ${ }^{7}$ throughout the experiment. They observed that processing safety messages increased the number of negative emotions felt by drivers. Among these, the intensity of anger felt by drivers correlated positively with an improvement in their VRU detection abilities. However, the authors were unable to conclude if the improvement in VRU detection performances was due to the emotion felt or to the information provided during the viewing of safety messages. In the present study, emotional elicitation was used to induce different intensities of anger in order to understand how a negative emotion might affect cyclist detection performances during driving. In this context, film clips, making no reference to road safety, and giving no information about VRU vulnerability, were used.

If anger alone, experienced when viewing the film clip, improves motorists' attentional abilities while driving, it could therefore be assumed that the effect of anger during driving depends on the intensity of anger felt. In this context, the detrimental effect observed in many studies (Garrity \& Demick, 2001; Jeon et al., 2015; Nesbit et al., 2007; Stephens et al., 2013) is likely to be linked to a high intensity of anger, as opposed to a moderate level of anger which could be beneficial for cyclist detection as suggested by Rogé's results . Consequently, we expected cyclist detection to be improved when a moderate intensity of anger was elicited, and a deterioration in cyclist detection ability when the intensity of anger was high. This assumption was called the anger intensity threshold hypothesis. In addition, as previously suggested by Stephens's eye-movement results, it could also be assumed that different visual scanning strategies would emerge across groups when individuals experienced different intensities of anger.

\footnotetext{
${ }^{7}$ (Scherer, 2005)
} 
As illustrated by some studies cited earlier, questionnaires and scales are commonly

151

152

153

154

155

156

157

158

159

160

161

162

163

164

165

166

167

168

169

170

171

172

used to assess emotional state. However, some works have shown that using a combination of self-reports, physiological data and behavioral measures is a very efficient way to infer mental processes (Smallwood \& Schooler, 2015) or emotional states (Valverde, Lera, \& Fernàndez, 2010). Hence, the question of how to best record reliable evidence of individuals' emotional state remains.

\subsection{Physiological assessment of emotional state}

According to Kreibig (2010), the study of the autonomic nervous system (ANS) is particularly relevant when investigating emotion. The ANS can be divided into two main branches: the parasympathetic nervous system which has an inhibitory effect on cardiac muscles through vagal efferent nerves, and the sympathetic nervous system which excites cardiac muscles via the norepinephrine neurotransmitter (Ruscio, Bos, \& Ciceri, 2017). Consequently, cardiac measures such as heart rate (HR), defined as the number of contractions of the heart per minute (bpm), and heart rate variability (HRV), which is the fluctuation in the time interval between heartbeats, are commonly used to determine which of the ANS components (i.e., sympathetic or parasympathetic) dominates. These measures therefore allow inferences to be made on the type of emotional processing used when individuals appraise stimuli or events (Kreibig, 2010; Niedenthal, Krauth-Gruber, \& Ric, 2006). According to Kreibig (2010), HR increase and overall HRV decrease, both reflecting sympathetic dominance, are usually observed when anger is experienced. However, HRV analysis regroups several domain methods (i.e., time, frequency and nonlinear) of RR interval series processing, and a number of indices can be calculated for each one of these. 
Some of these are used often, especially the standard deviation of the average NN interval ${ }^{8}$ calculated over short periods (SDANN), and pNN50 which is the proportion of interval differences of successive $\mathrm{NN}$ intervals greater than $50 \mathrm{~ms}$, both related to the time domain method. Power spectral density collected for high frequency band (HF) and low frequency band (LF) of interval tachograms are also often used as frequency domain indexes. The nonlinear domain is used less, probably because processing of some of the indexes is complicated. Nevertheless, because interpretation of their results is relatively easy, some indexes are worth processing (Tarvainen, 2014). In this context, we only focused on the Poincaré plot which is a graphical representation of the correlation between successive RR intervals. Two indexes (SD1 and SD2) can then be calculated, along with sample entropy (SampEn) which is a reliable estimator of the complexity of a signal especially for short time series (<200points) (Riganello, Cortese, Arcuri, Quintieri, \& Dolce, 2015).

All these indexes either provide information about short-term variability in cardiac rhythm (e.g., pNN50, SD1, HF), or describe long-term variability (SDANN, SD2, LF) or signal complexity (e.g., SampEn). While long-term variability in cardiac rhythm tends to represent the sympathetic branch of the ANS, short-term variability refers to parasympathetic dominance (Task Force of The European Society of Cardiology and The North American Society of Pacing and Electrophysiology, 1996). In addition, according to Valenza, Allegrini, Lanatà and Scilingo (2012), rest is characterized by chaotic behavior dynamics that cause entropy increase. By contrast, entropy tends to decrease with arousal increase. As arousal variations broadly correlate with ANS activity (Salvia, 2012), entropy variations could thus provide information about ANS activity.

\footnotetext{
${ }^{8}$ (i.e., the interval from one $\mathrm{R}$ peak to the subsequent $\mathrm{R}$ peak)
} 
In the literature on cardiovascular indexes of HRV related to anger, Francis, Penglis and McDonald (2016) found a significant increase in SDNN values after an anger inducing task. In addition, Kop et al. (2011) found that the level of frustration in an anger recall task tended to be associated with higher LF values. Marci, Glick, Loh and Dougherty (2007) also found a significant decrease in HF values in participants who had to listen to anger scripts while trying to imagine the event portrayed as vividly as possible. These studies all illustrate sympathetic dominance when anger is experienced. However, cardiac measurements are not the only way to assess ANS activity.

Some studies have revealed a relationship between pupillary size, the ANS and emotions. While pupil dilation is mediated by the sympathetic system, pupil contraction is under parasympathetic system control (Sirois \& Brisson, 2014). Emotional arousal is associated with pupil size variations. Emotions with a high arousal (e.g., anger) tend to increase pupil size (Bradley, Miccoli, Escrig, \& Lang, 2008). In addition, according to Okutucu et al. (2016), there are a number of correlations between dynamic pupillometry (i.e., a set of different metrics related to pupil dilation and contraction as well as velocity and duration of pupil contraction and dilation) and some of the previously cited indexes of HRV (e.g., SDANN, pNN50, HF and LF/HF). Consequently, links can also be made between autonomic functions assessed by pupil and cardiac measures. To the best of our knowledge, no study couples cardiac (HR and HRV) and pupillary data in order to assess different intensities of anger.

In the light of previous studies, it was assumed that the sympathetic branch of ANS would dominate an individual's overall autonomic nervous activity when anger was experienced. This phenomenon would be further amplified as the intensity of anger elicited became stronger. This assumption was named the sympathetic dominance hypothesis. 


\section{Method}

\subsection{Participants}

Forty-five participants (18 males, 27 females), aged between 19 and 42 years $(M=28.5 ; S D=5.8)$ took part in this experiment. They all had at least two years of driving experience. On average, they used their car four times a week and covered more than 150 kilometers per week. All participants reported normal or corrected-to-normal vision and audition.

\subsection{Material}

\subsubsection{Film clips}

Three film clips retrieved from a previous study (Schaefer, Nils, Sanchez, \& Philippot, 2010) were selected on the basis of their propensity to elicit a neutral state and two levels of anger. For the anger-inducing film clips, the selection was based firstly on a set of 10 film clips with high anger discreetness scores (i.e., mean score obtained on the anger item of the Differential Emotions Scale (DES) ${ }^{9}$, minus mean scores obtained on all remaining items of the DES). Two of the film clips with the highest anger discreetness scores (i.e., film clips with the highest propensity to elicit anger) were then selected on the basis of their mean anger scores on the DES. In this way, we chose two film clips that potentially elicited anger at different levels: slight (no. 30 in the Schaefer's baseline with an anger score of 3.16) and strong (no. 2 with an anger score of 5.04). For the neutral film clip, we selected a clip (no. 49) with a very low level on the anger item of DES (anger score of 1). Precautions were also taken regarding this clip,

${ }^{9}($ Izard, Dougherty, Bloxom, \& Kotsch, 1974) 
for which no other item of DES exceeded a score of 1, thus ensuring that the film clip could be considered as neutral.

The neutral film clip showed a woman walking along the street in a market-place shopping area. The film clip inducing slight anger showed a man undergoing intense interrogation, and the film clip inducing strong anger showed a man randomly shooting people from his balcony. Because the film clips were of different lengths, we made changes to obtain three comparable videos (mean length $=1 \mathrm{mn} 55 \mathrm{~s} ; \mathrm{SD}=10 \mathrm{~s}$ ).

\subsubsection{Emotional wheel (EW)}

To measure the intensity of anger felt by participants, we used the Emotional Wheel (EW) (Rogé et al., 2015). This tool is a visual analogue scale from which the intensity of anger can be extracted and converted into a percentage.

\subsubsection{Apparatus}

Driving simulator. A car-driving simulator (Figure 1) was used to reproduce the same driving conditions for all participants, and ensured their absolute safety. It consists of a Peugeot 308 car cabin equipped with a video projector set behind each of the five screens (size: $2.25 \mathrm{~m} \times 8.25 \mathrm{~m}$ at a horizontal visual angle of $180^{\circ}$ ) used for the road scene ahead, and one screen (size: $2.25 \mathrm{~m} \times 3.00 \mathrm{~m}$ ) behind the cabin for the inside rear view, with a video projector hung from the ceiling. Two monitors (size: $.56 \mathrm{~m} \mathrm{x} \mathrm{.48} \mathrm{m)} \mathrm{installed} \mathrm{on} \mathrm{both} \mathrm{sides} \mathrm{of} \mathrm{the} \mathrm{cabin}$ at the same height as exterior flat mirrors (at a distance of $1.08 \mathrm{~m}$ from the mirrors) enabled participants to monitor the road environment to the side of, and behind their car. Road images were projected at an average refresh rate of $59 \mathrm{~Hz}$, and driving data were recorded at the same 
frequency. Traffic and car engine sounds were played through a wooden subwoofer and two satellite speakers placed on each side of the participant.

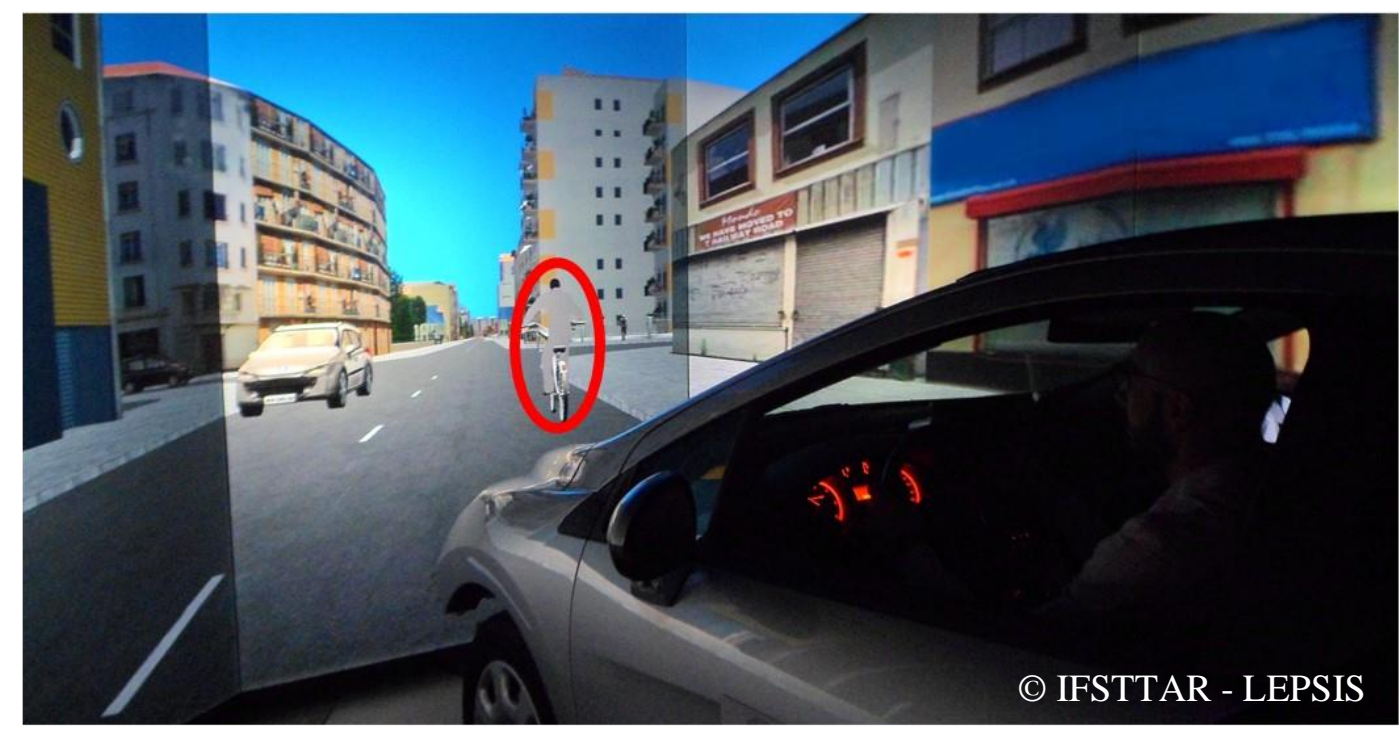

Figure 1. The OSG-Sim² simulator in the LEPSIS unit at IFSTTAR including an example of the type of cyclist (circled in red) participants had to detect

Electrocardiogram. A Bionomadix transmitter (BIOPAC Systems Inc.) allowing wireless connection with an MP150 data recording system (BIOPAC Systems Inc.) was used to collect cardiac signals. Electrocardiogram (ECG) was continuously recorded and sampled at $1000 \mathrm{~Hz}$ from the cardiac baselines until the end of the experiment. The ECG was analog lowpass $(.05 \mathrm{~Hz})$ and high-pass $(35 \mathrm{~Hz})$ filtered at acquisition, as an approximately $5-30 \mathrm{~Hz}$ range covers most of the frequency content of QRS complex (Pahlm \& Sörnmo, 1984). Triggers, which were manually positioned during the recording to define the beginning and end of the baseline, and of the film clip session, were automatically sent by the simulator to the ECG at the beginning and end of the driving session to ensure accurate synchronization. 
Eye tracking. The Mobile Tobii Glasses Eye-Tracker was used to continuously record participants' eye position from when film clip viewing began until the end of the experiment. The glasses provided video-based eye tracking using the dark pupil and corneal reflections and the embedded scene camera operated at $30 \mathrm{~Hz}$ with $56^{\circ}$ x $40^{\circ}$ scene coverage.

\subsection{Procedure}

The experiment began with several tests (e.g., Monoyer, Parinaud, Ishihara, and Useful Visual Field) in order to measure individuals' visual acuity (from near and far), their ability to discriminate colors, and the size of their visual field respectively.

Three $\mathrm{Ag}-\mathrm{AgCl}$ pre-gelled electrodes were then attached to the participants following a modified lead II configuration. Electrodes were connected to the Bionomadix transmitter (BIOPAC Systems Inc.) to record participants' cardiac activity. After a few seconds of recording to ensure the equipment was functioning correctly, participants were seated comfortably in the driving simulator. They then carried out a driving training session in an urban environment. They were also asked to respect the Highway Code and to detect VRUs without making any mistakes. In order to do this, they had to activate the headlight lever behind the steering wheel as soon as they detected pedestrians or cyclists. These training sessions allowed participants to become familiar with the simulator and to accustom themselves to the lever used to indicate detection of VRUs.

Participants were then equipped with the eye-tracker outside the car driving simulator. A system-guided 9-point calibration was performed until the highest possible tracking quality was achieved. Participants returned to the driving simulator, were comfortably seated and were asked to relax for $5 \mathrm{~min}$ in order to record cardiac baseline. This $5 \mathrm{~min}$ rest session was divided in two: $2 \min 30 \mathrm{~s}$ with their eyes closed and $2 \min 30 \mathrm{~s}$ with their eyes open. The closed-eye 
period allowed individuals to calm down, and the open-eye period was considered as the cardiac baseline standard.

They then watched one of the three film clips on a large screen (visual angle of $31^{\circ} \mathrm{x} 17^{\circ}$ ) while still sitting in the simulator. The sound track was played through speakers positioned behind the participant in order to create an immersive watching situation. Participants were randomly assigned to one of the three experimental conditions. Before and after viewing the film clip as well as after the driving session, participants were asked to assess the highest emotional intensities of anger they felt throughout each stage respectively.

Finally, participants carried out a second driving task which lasted approximately five minutes. They were instructed, as during training, to respect the Highway Code and to detect VRUs (10 pedestrians and 5 cyclists) in an urban environment as quickly as possible without making any mistakes. It should be noted that only cyclist detections were analyzed. Pedestrians were used as decoys to orient the attention of participants towards the whole road scene, and to distract them from the real targets (i.e., cyclists), as might be the case when driving in a real environment. When driving was completed, participants had to complete the EW. The total experiment lasted about an hour and a half.

\subsection{Measures}

Subjective intensity of anger was assessed using the Emotional Wheel (EW). Data collected from this EW were recorded as lengths (in $\mathrm{mm}$ ) between the beginning of each segment of the wheel and the marks drawn on it by participants.

For cardiac data, each participant's filtered cardiac signal was visually checked to correct any artifacts (see Berntson et al., 1997; Berntson \& Stowell, 1998) for artifact correction 
methods used). R peaks were detected on cardiac signal in order to calculate RR intervals corresponding to the time between two R peaks. Several indexes were then calculated with RR interval series using Kubios HRV software v.2.2. Heart Rate was computed in beats per minute (b.p.m.).

We also computed a number of indexes obtained from HRV analysis. SDANN and pNN50, were calculated first. An LF/HF ratio was also considered. This index gives information about how power (i.e., RR interval variance) distributes as a function of frequency. LF/HF ratio allows the partial inference of which ANS branch dominates. While LF ranging from $.04 \mathrm{~Hz}$ to $.15 \mathrm{~Hz}$ represent long term variability which is a marker related to sympathetic activity, $\mathrm{HF}$ ranging from $.15 \mathrm{~Hz}$ to $.4 \mathrm{~Hz}$ represent short term variability and are related to parasympathetic activity.

In addition, a Poincaré plot, providing a graphic display of the correlations between successive RR intervals (i.e., plot of $R R j+1$ as a function of $R R j$ ) was also computed. Standard deviation of the points perpendicular to the line of identity denoted by SD1 and SD2 were analyzed in order to parameterize the shape's ellipse formed by cloud points describing short and long term variability in cardiac rhythm respectively.

Sample entropy (SampEn) was computed through complex calculations of differences between RR interval series in order to quantify the extent of signal entropy, in other words, how anarchic the signal is (See Riganello et al., 2015; Tarvainen, 2014) for more information about how SampEn is calculated).

Pupil size was estimated through an index. A value was calculated for each timestamp corresponding either to a pupil dilation (i.e., >100) or to a pupil contraction (i.e., <100) with one hundred corresponding to the average value of the pupil during the calibration. By doing this, we were able to avoid inherent individual differences. 
The delay between the first hit on cyclists and use of headlight lever (time for cyclist detection) was computed as cyclist detection performance. The length of the first glance at the cyclist (first glance length) was also calculated. Both metrics were expressed in milliseconds. Finally, saccadic amplitudes (in degrees) were also computed to evaluate the ocular strategies implemented by individuals during driving.

On the basis of the sympathetic dominance hypothesis, HR and pupillary size increase combined with an increase in long-term variability indexes of HRV and a decrease in shortterm indexes was expected for both groups exposed to anger eliciting film clips compared to those exposed to neutral film clips. This phenomenon was expected to appear at least during the film clip watching stage when the effect of emotional induction was supposed to be still present. This pattern would also be more salient in the strong anger group than in the slight anger group, in which it would be more pronounced than in the neutral group.

In line with the anger intensity threshold hypothesis, the times for cyclist detection and of first glance length in slight anger group participants was expected to be shorter than those observed in the strong anger and neutral groups. Saccadic amplitude was explored in order to find out whether specific visual scanning strategies emerged between the different groups.

\subsection{Data processing}

The assumptions underlying the ANOVAs were checked using the KolmogorovSmirnov test for normal distribution and Levene's test for variance homogeneity for all the following analyses. Comparisons of means using a post-hoc Fisher LSD test were conducted when significant differences appeared following ANOVAs. Means were considered as significantly different when the probability of a Type 1 error was less than or equal to .05 . Mann-Whitney tests were conducted to compare groups when non-parametric tests were used. 
For subjective data, a Kruskal-Wallis test was used due to non-normal distribution of anger intensity prior to watching the film clip. An ANOVA was then performed on intensities of anger felt after the film clip had been watched, with film clip (neutral vs. slight anger vs. strong anger) as a between-subjects factor. Friedman's ANOVA was also conducted on intensities of anger felt throughout the study, with stages (baseline vs. induction vs. driving) as a within-subject factor. Wilcoxon tests were performed when significant differences appeared following Friedman's ANOVA.

ANOVAs were conducted on cardiac data for HR, SDANN, pNN50, LF/HF ratio, SD1, SD2 and SampEn at baseline (i.e. open eyes), for HR ratio (described below), pNN50, LF/HF ratio, SD1 and SampEn during film clip viewing and for HR ratio, SDANN, pNN50, SD1, SD2, SampEn during the driving stage, with film clip as a between-subjects factor (neutral vs. slight anger vs. strong anger). By contrast, Kruskal-Wallis tests were performed for SDANN and SD2 during the film clip stage and for LF/HF ratio during the driving stage.

For ocular data, an ANOVA was conducted on pupil size for both film clip and driving sessions, with film clip as between-subjects factor (neutral vs. slight anger vs. strong anger). In addition, ANOVAs were performed for cyclist detection times, for first glance length and saccadic amplitudes, and film clip was once again used as a between-subjects factor (neutral vs. slight anger vs. strong anger).

\section{Results}

\subsection{Emotional assessment using subjective data}

Results did not show any significant differences between groups regarding the assessment of anger experienced before watching the film clips $(H(2,44)=.64, p=.73)$. The intensity of anger felt by the three groups was therefore comparable before watching the film. 
Significant differences appeared between groups in the anger assessments following film clip viewing $\left(F(2,42)=31.12, p=.001, \eta p^{2}=.597\right)$. LSD post hoc tests revealed that all intensities

401 of anger significantly differed between the three groups $(p<.01)$. The neutral film clip triggered 402 the lowest level of anger $(M=1.31, S D=1.70)$, the slight anger clip a moderate level of anger $403(M=24.12, S D=13.11)$, and the strong anger clip was rated with the highest intensity 404 $(M=37.13, S D=15.44)$ (Figure 2). Self-reported assessment therefore supported the efficiency of the emotional induction.

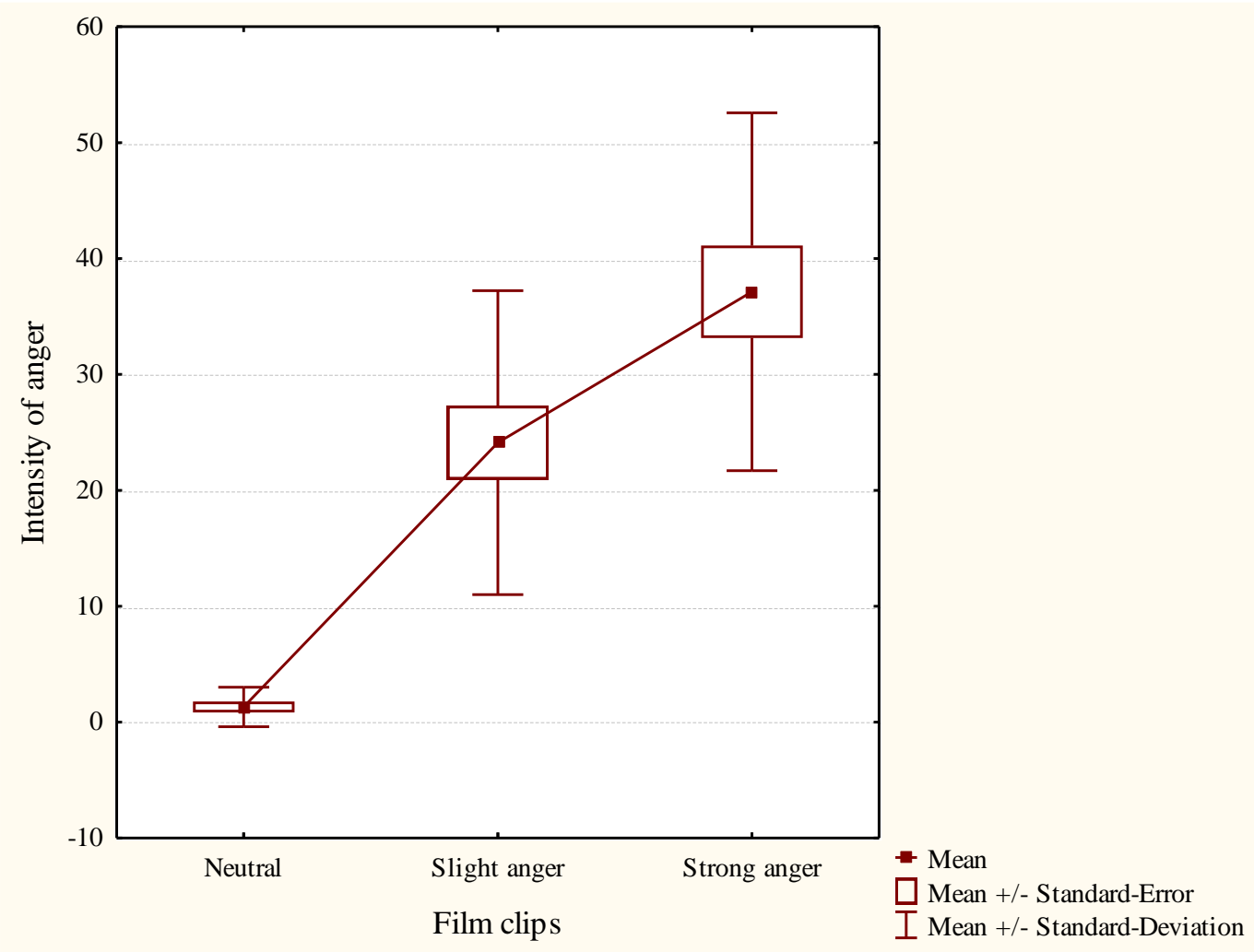


413 of anger significantly differed between the stages $(\mathrm{p}<.001)$ describing an increase of anger 414 intensity between the baseline $(M=1.27, S D=1.70)$ and the induction stage $415(M=21.87, S D=18.59)$ and a decrease between the induction and the driving session $416(M=7.87, S D=11.74)$ (Figure 3). It should be noted that the intensity of anger for the driving 417 session was significantly higher than those obtained during the baseline session.

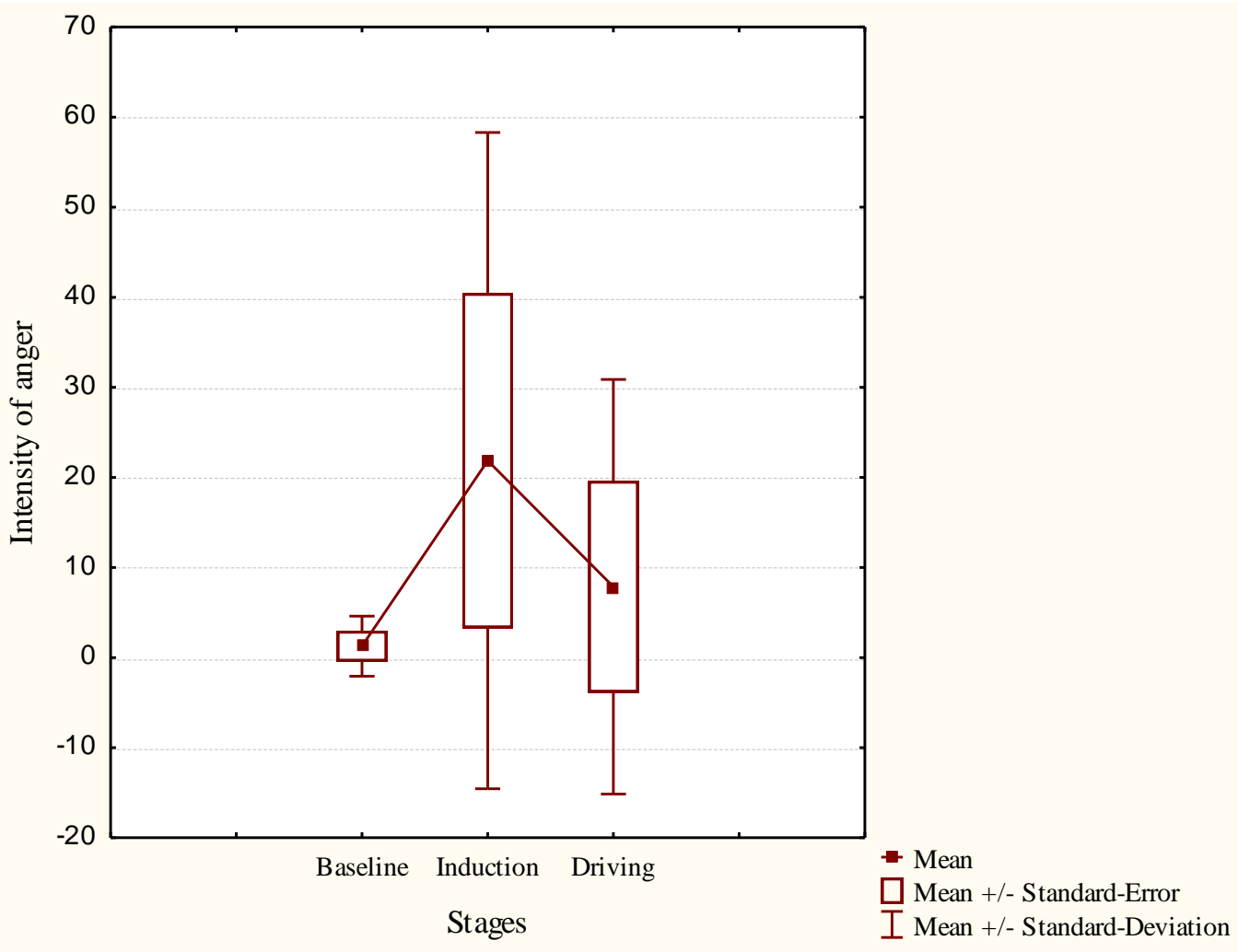




\subsection{Emotional assessment using cardiac data}

\subsubsection{Baseline}

Results at baseline did not show significant differences between groups for any cardiac metric values $(p \geq .10)$ except for $\operatorname{HR}\left(F(2,42)=5.05, p=.01, \eta p^{2}=.19\right)$. We therefore decided to compensate the effect of individual differences in subsequent analyses related to HR. To this end, a ratio was used dividing the HR values collected during the film clip watching stage and during the driving session respectively by HR values recorded at baseline.

\subsubsection{During film clip watching}

Results showed a significant effect of film clip $\left(F(2,42)=3.37, p=.04, \eta p^{2}=.14\right.$, $\mathrm{CI}=[.05, .08])$ on HR ratio. Significant differences between the neutral group $(M=1.02, \mathrm{SD}=.04)$ and strong anger groups $(M=.96, \mathrm{SD}=.04)$ as well as a limited effect $(p=.06)$ between the neutral and slight anger groups $(M=.98, \mathrm{SD}=.08)$ were observed, indicating that anger-eliciting film clips triggered lower HR ratio values than the neutral film clip.

Analysis of pNN50 revealed significant differences between groups $\left(F(2,42)=4.23, p=.02, \eta p^{2}=.17, \mathrm{CI}=[27.05,41.60]\right)$, with lower values for the neutral group $(M=18.94, S D=16.14)$ than for the slight anger group $(M=40.63, S D=26.63)$ and the strong anger group $(M=40.52, S D=22.36)$.

Results for the LF/HF ratio highlighted a significant effect of film clip $\left(F(2,42)=3.45, p=.04, \eta p^{2}=.14, \mathrm{CI}=[1.02,1.83]\right)$, showing significant differences between the neutral group $(M=2.21, S D=1.91)$ and the slight anger group $(M=1.15, S D=.86)$, and between the neutral and strong anger groups $(M=1.07, S D=.91)$. 
Film clips had a limited effect on SampEn $(p=.09)$, in the neutral group

447

448

449

450

451

452

453

454

455

456

457

458

459

460

461

462

463

464

465

466

467

468

469

\subsubsection{During the driving session}

A significant effect of film clip on pNN50 was observed $\left(F(2,42)=3.63, p=.04, \eta p^{2}=.15, \mathrm{CI}=[21.25,33.09]\right)$, with lower values for the neutral group $(M=15.47, S D=10.70)$ than for the slight anger group $(M=31.30, S D=21.76)$ and the strong anger group $(M=32.63, S D=20.03)$.

However, film clips had a limited effect on LF/HF ratio $(p=.08)$, in the neutral group $(M=2.90, S D=1.83), \quad$ slight anger $(M=2.30, S D=1.94)$, and strong anger group $(M=1.55, S D=.82)$.

The same limited effect was observed for SD1 $(p=.06)$ in the neutral group $(M=25.09, S D=8.94)$, slight anger $(M=39.18, S D=19.57)$, and strong anger group $(M=42.69, S D=25.95)$.

Regarding SampEn, a significant effect of film clip was found between groups $\left(F(2,42)=3.49, p=.04, \eta p^{2}=.14, \mathrm{CI}=[1.56,1.72]\right)$, with significant differences between the neutral group $(M=1.48, S D=.31)$ and the slight anger group $(M=1.70, S D=.26)$, and between the strong anger group $(M=1.71, S D=.20)$ and the neutral group. Film clips had no significant effect on the following cardiac indexes: HR ratio, SDANN, and SD2.

Overall, analysis of cardiac data highlighted evidence of comparable physiological patterns during film clip viewing and during the driving task (see Table 1). Interestingly, both anger conditions differed from the neutral condition in almost all cases. However, the observed 
variations, broadly describing short term variability dominance for anger groups, were

471 unexpected and contradictory to the sympathetic dominance hypothesis.

472

473 Table 1

Summary table of cardiac metrics variations throughout the different stages of the experiment

\begin{tabular}{lcc}
\hline Cardiac indexes & Film clip watching & Driving \\
\hline HR ratio & Neutral $>$ Strong anger & $\mathrm{X}$ \\
& $($ Neutral $>$ Slight anger $)$ & $\mathrm{X}$ \\
SDANN & $\mathrm{X}$ & Neutral $<$ Slight anger \& Strong anger \\
pNN50 & Neutral $<$ Slight anger \& Strong anger & (Neutral $>$ Strong anger) \\
LF/HF ratio & Neutral $>$ Slight anger \& Strong anger & (Neutral < Slight anger \& Strong anger) \\
SD1 & X & X \\
SD2 & X & Neutral $<$ Slight anger \& Strong anger \\
SampEn & &
\end{tabular}

$475 \mathrm{X}$ No significant differences were observed between groups

476 () Result based on limited effects

477

\subsection{Emotional assessment using eye pupil size data}

Results during film clip viewing firstly revealed a significant effect of film clip on pupil $\operatorname{size}\left(F(2,42)=4.61, p=.02, \eta p^{2}=.18, \mathrm{CI}=[96.29,102.98]\right)$, with significantly higher values

481 for the neutral group $(M=104.34, S D=10.72)$ than for the values recorded for the strong anger 482 group $(M=93.20, S D=6.98)$. In addition, significantly higher values were observed in the 483 slight anger group $(M=101.72, S D=12.29)$ than in the strong anger group. However, no 484 significant effect of film clip was found during the driving stage in the three groups. 
Overall, the results regarding pupil size highlighted the emergence of a specific pattern during the film clip stage. This pattern consisted of smaller pupil sizes in the strong anger group only. This pattern was therefore not consistent with the sympathetic dominance hypothesis and did not seem to be maintained during the driving session.

\subsection{Cyclist detection}

Results did not emphasize any significant effect of film clip either for cyclist detection time $(F(2,41)=.24, p=.79)$ or for first glance length $(F(2,42)=1.11, p=.34)$. This did not support the anger intensity threshold hypothesis, and suggested that the emotional state induced in this experiment did not affect VRU detection performances. Nevertheless, further investigations needed to be carried out to determine whether emotional state affected background processes such as visual scanning strategies while driving.

\subsection{Visual scanning strategies}

Even though results did not show a significant effect of the film clip variable on first glance length and cyclist detection time, a significant effect of film clip was observed on saccadic amplitudes $\left(F(2,42)=4.98, p=.01, \eta p^{2}=.19, \mathrm{CI}=[1.37,1.59]\right)$ with significantly larger saccades for the neutral group $(M=1.70, S D=.37)$ than for the strong anger group $(M=1.30, S D=.33)$. A trend $(p=.06)$ towards larger saccades in the neutral group than in the slight anger group $(M=1.46, S D=.31)$ also emerged (Figure 4). These results suggested that groups did not use the same visual scanning strategies. This applied particularly to the anger groups, particularly the strong anger group, whose ocular behavior when driving consisted of smaller saccadic amplitudes. 


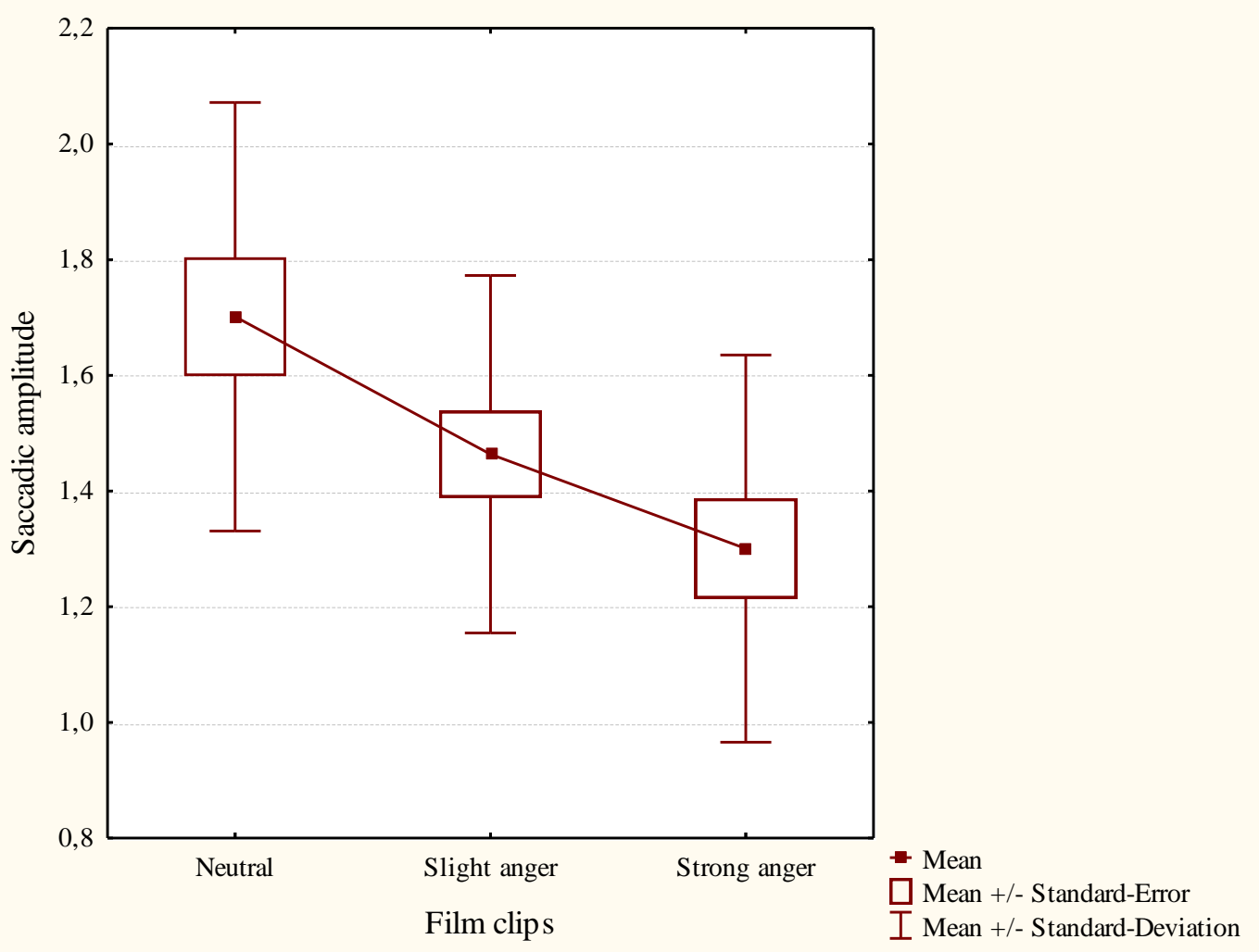

Figure 4. Saccadic amplitudes observed during the driving stage for the three groups

\section{Discussion}

The aim of the present research was to investigate the role of anger in cyclist detection.

Results for the film clip watching stage revealed that film clips elicited three distinct intensities of anger according to self-report. In addition, physiological data (cardiac and ocular) allowed us to differentiate the neutral group from the anger groups.

It should be remembered that, while SDANN and SD2 refer to long-term variability associated with sympathetic activity, pNN50 and SD1 are related to short term variability

518 associated with parasympathetic activity. In addition, the LF/HF ratio provides a good 519 illustration of the balance between short and long term variability (Tarvainen, 2014; Task Force of The European Society of Cardiology and The North American Society of Pacing and 
While pupil dilation seems to be linked to the sympathetic system, decrease in pupil size appears to be mediated by the parasympathetic system (Sirois \& Brisson, 2014).

In the present study, we found lower values of HR and LF/HF ratios, and higher values of pNN50 combined with lower pupil size in participants who watched the anger film clips than in those who watched the neutral film. Consequently, parasympathetic dominance can be assumed during this stage for both anger groups. This is inconsistent with the sympathetic dominance hypothesis. However, the question of what parasympathetic dominance means in such a context (i.e., video watching) remains.

According to Luce, Payne and Bettman (1999) high levels of negative emotions induce regulation strategies such as avoidance coping. Furthermore, other authors have highlighted physiological patterns, especially cardiac patterns, describing this emotion regulation phenomenon. Denson, Grisham and Moulds (2011), for example, asked three groups of participants to watch an anger-inducing video. In one group (control), participants only had to watch the video. In the second group (suppression), they had to try to control their facial expressions and to behave in such a way that a person watching them would not know what they were feeling. In the last group (reappraisal), participants had to manage their emotional reactions; they were asked to try to maintain a neutral mood and not to be overwhelmed by the anger elicited by the video. Researchers observed a significant increase in short-term variability and decreased HR values among participants in the reappraisal condition. Similar types of pattern were also observed in studies using pictures to induce a negative emotional state (Kuoppa, Tarvainen, Karhunen, \& Narvainen, 2016; Sarlo, Palomba, Buodo, Minghetti, \& Stegagno, 2005).

If Luce's rationale is followed, the existence of an anger threshold predicting the establishment of reappraisal strategies seems feasible. In other words, in our study, individuals exposed to the strong anger film clip could be expected to employ reappraisal strategies in order 
to deal with bad feelings. However, since differences between groups were only observed for both anger groups when compared to the neutral group, it can be assumed that this threshold might be located below the intensity of anger elicited by the slight anger film clip. These results suggest that both anger film clips elicited an excessively high intensity of anger, which, in turn, drove individuals to implement reappraisal strategies to avoid being overwhelmed by negative feelings.

Results obtained from self-report revealed high levels of anger for groups exposed to the anger film clips. This result could be interpreted as an inefficient way of coping. Nonetheless, the EW allows us to assess the extent to which individuals are affected by emotions while watching the film clips without taking account of how people react when faced with these emotions. One explanation could be that individuals were strongly affected by the film clip at a particular moment, as shown by anger intensity scores collected from EW. They then resorted to reappraisal strategies.

During the driving stage, a comparable physiological pattern also appeared for anger groups when compared to the neutral group. pNN50 values tend to depict a dominance of parasympathetic activity in individuals who watched one of the anger eliciting film clips. In addition, SampEn values were significantly higher in both anger groups than in the neutral group. According to Valenza et al. (2012), rest is characterized by chaotic behavior dynamics manifested by increased entropy, which tends to decrease with arousal increase. As the parasympathetic branch of the ANS is involved not only in rest states but also when reappraisal strategies have been set up, the significantly higher value for SampEn when participants were watching the anger film clips is congruent with an emotion-regulation strategy. Besides, selfreport revealed lower intensities of anger for all groups for the driving session when compared to the induction stage. This result would either suggest that emotion-regulation strategies have 
571 been effectively implemented for anger groups throughout the driving, or anger simply

572 decreased due to the attentional focus on the VRU detection task.

573

It should also be noted that we did not find exactly the same physiological results for the film clip stage and during driving. One interpretation could be that unlike during the filmclip viewing stage, individuals had to drive during the driving session. In this context, cognitive processes such as attention management and motor activity are required. According to Porges et al. (2007), motor activity is strongly related to ANS variations. Therefore, it is feasible that driving affected the physiological variables studied. However, can it be assumed that the pattern observed reflects only reappraisal strategies affected by driving activity?

According to Beauchaine (2001); Griffiths et al. (2017), sustained attention is accompanied by parasympathetic dominance. Therefore, because of the instructions given to participants during the driving task (i.e., to detect VRUs), it is possible that emotional elicitation and implementing coping strategies affected the way that individuals subsequently managed their attention to the targets, and became more attentive and more efficient in detecting VRUs.

When VRU detection performance was studied, there was no evidence to suggest that emotion had affected this performance, since the film clips did not affect cyclist detection times. However, on further investigation of ocular behaviors, results showed significant differences in saccadic amplitudes between groups. Anger groups made smaller saccades than participants in the neutral group. This result suggests that individuals in the anger groups adopted a specific attention management strategy.

There are at least two explanations for this phenomenon. On one hand, it can be assumed that anger-provoked people were in an optimal attention state as previously described. In this case, their attentional management would be more efficient. In other words, they would probably focus their attention more quickly on relevant space areas (i.e., cyclists they had to 
detect). On the other hand, according to Mackenzie and Harris (2017), cognitive load could

596 impair visual scanning during driving. Consequently, it is also feasible that the conservation of

597 the coping strategies used during driving previously mentioned carried a cognitive cost, which,

598 in turn, manifested itself in smaller saccadic amplitudes. Future studies would be needed to

599 investigate this issue using, for example, assessment of cognitive load through (NASA, 1986)

600 or more quantitative measures such as functional near-infrared spectroscopy (fNIRS).

601

602

603

Overall, these results demonstrated that both cardiac measures and pupil size provide interesting evidence of emotional state and allow diagnosis of the implementation of reappraisal strategies. This research also raises some issues about the relevance of focusing on discrete emotions. Appraisal approaches of emotion combined with physiological measurement seem to be a better way of taking into account how individuals deal with emotional events. In addition, there was no significant difference between groups regarding detection performances. This point suggests that emotion, alone, in the absence of any reference to the vulnerability of certain road users, was not sufficient here to improve VRU detection abilities. In addition, the intensities of anger manipulated in this study did not replicate the beneficial effect on VRU detection performances while driving found by Rogé et al. (2015). The next step will be to combine vulnerability information with emotion in film clips used to elicit negative emotions, to determine whether or not this can improve cyclist detection performance during driving. This will help to provide guidelines on the most effective way of communicating in order to improve VRU detection abilities in motorists.

\section{Acknowledgements}




\section{References}

Beauchaine, T. (2001). Vagal tone, development, and Gray's motivational theory: Toward an integrated model of autonomic nervous system functioning in psychopathology. Development and Psychopathology, 13(2), 183-214.

Berntson, G. G., \& Stowell, J. R. (1998). ECG artifacts and heart period variability: don't miss a beat! Psychophysiology, 35(1), 127-132.

Berntson, G. G., Thomas Bigger, J., Eckberg, D. L., Grossman, P., Kaufmann, P. G., Malik, M., ... Van Der Molen, M. W. (1997). Heart rate variability: Origins, methods, and interpretive caveats. Psychophysiology, 34(6), 623-648.

Bouaoun, L., Haddak, M. M., \& Amoros, E. (2015). Road crash fatality rates in France: A comparison of road user types, taking account of travel practices. Accident Analysis \& Prevention, 75, 217-225. https://doi.org/10.1016/j.aap.2014.10.025

Bradley, M. M., Miccoli, L., Escrig, M. A., \& Lang, P. J. (2008). The pupil as a measure of emotional arousal and autonomic activation. Psychophysiology, 45(4), 602-607. https://doi.org/10.1111/j.1469-8986.2008.00654.x

Callejas, A., Lupianez, J., Funes, M. J., \& Tudela, P. (2005). Modulations among the alerting, orienting and executive control networks. Experimental Brain Research, 167(1), 27-37. https://doi.org/10.1007/s00221-005-2365-z

Costa, P. T., \& McCrae, R. R. (1992). Revised NEO personality inventory (NEO PI-R) and NEO five factor inventory (NEOFFI). Odessa, FL: Psychological Assessment Resources, Inc.

Cheung, S. Y., \& Lam, E. T. C. (2005). An Innovative Shortened Bilingual Version of the Profile of Mood States (POMS-SBV). School Psychology International, 26(1), 121128. https://doi.org/10.1177/0143034305050898 
Denson, T. F., Grisham, J. R., \& Moulds, M. L. (2011). Cognitive reappraisal increases heart rate variability in response to an anger provocation. Motivation and Emotion, 35(1), 1422.

Ellis, H. C., \& Moore, B. A. (2005). Mood and Memory. In T. Dalgleish \& M. J. Power (Eds.), Handbook of Cognition and Emotion (pp. 193-210). Chichester, UK: John Wiley \& Sons, Ltd. https://doi.org/10.1002/0470013494.ch10

Engel, F. L. (1971). Visual conspicuity, directed attention and retinal locus. Vision Research, $11(6), 563-576$.

Francis, H. M., Penglis, K. M., \& McDonald, S. (2016). Manipulation of heart rate variability can modify response to anger-inducing stimuli. Social Neuroscience, 11(5), 545-552. https://doi.org/10.1080/17470919.2015.1115777

Garrity, R. D., \& Demick, J. (2001). Relations Among Personality Traits, Mood States, and Driving Behaviors. Journal of Adult Development, 8(2), 109-118. https://doi.org/10.1023/A:1026446002317

Griffiths, K. R., Quintana, D. S., Hermens, D. F., Spooner, C., Tsang, T. W., Clarke, S., \& Kohn, M. R. (2017). Sustained attention and heart rate variability in children and adolescents with ADHD. Biological Psychology, 124, 11-20. https://doi.org/10.1016/j.biopsycho.2017.01.004

Hancock, P. A., Wulf, G., Thom, D., \& Fassnacht, P. (1990). Driver workload during differing driving maneuvers. Accident Analysis \& Prevention, 22(3), 281-290. https://doi.org/10.1016/0001-4575(90)90019-H

Hole, G. J., Tyrrell, L., \& Langham, M. (1996). Some factors affecting motorcyclists' conspicuity.

Ergonomics, $39(7)$ 946-965. https://doi.org/10.1080/00140139608964516 
Izard, C. E., Dougherty, F. E., Bloxom, B. M., \& Kotsch, N. E. (1974). The Differential Emotions Scale: A method of measuring the subjective experience of discrete emotions. Nashville: Vanderbilt University, Department of Psychology.

Jeon, M., Walker, B. N., \& Gable, T. M. (2015). The effects of social interactions with invehicle agents on a driver's anger level, driving performance, situation awareness, and perceived workload. Applied Ergonomics, 50, 185-199. https://doi.org/10.1016/j.apergo.2015.03.015

Jeon, M., Walker, B. N., \& Yim, J.-B. (2014). Effects of specific emotions on subjective judgment, driving performance, and perceived workload. Transportation Research Part F: Traffic Psychology and Behaviour, 24, 197-209. https://doi.org/10.1016/j.trf.2014.04.003

Kop, W. J., Synowski, S. J., Newell, M. E., Schmidt, L. A., Waldstein, S. R., \& Fox, N. A. (2011). Autonomic nervous system reactivity to positive and negative mood induction: The role of acute psychological responses and frontal electrocortical activity. Biological Psychology, 86(3), 230-238. https://doi.org/10.1016/j.biopsycho.2010.12.003

Kreibig, S. D. (2010). Autonomic nervous system activity in emotion: A review. Biological Psychology, 84(3), 394-421. https://doi.org/10.1016/j.biopsycho.2010.03.010

Kuoppa, P., Tarvainen, M. P., Karhunen, L., \& Narvainen, J. (2016). Heart rate reactivity associated to positive and negative food and non-food visual stimuli (pp. 5279-5282). IEEE. https://doi.org/10.1109/EMBC.2016.7591918

Luce, M. F., Payne, J. W., \& Bettman, J. R. (1999). Emotional Trade-Off Difficulty and Choice. Journal of Marketing Research, 36(2), 143. https://doi.org/10.2307/3152089

Mackenzie, A. K., \& Harris, J. M. (2017). A link between attentional function, effective eye movements, and driving ability. Journal of Experimental Psychology: Human Perception and Performance, 43(2), 381-394. https://doi.org/10.1037/xhp0000297 
Magazzu, D., Comelli, M., \& Marinoni, A. (2006). Are car drivers holding a motorcycle licence less responsible for motorcycle?Car crash occurrence? Accident Analysis \& Prevention, 38(2), 365-370. https://doi.org/10.1016/j.aap.2005.10.007

Marci, C. D., Glick, D. M., Loh, R., \& Dougherty, D. D. (2007). Autonomic and prefrontal cortex responses to autobiographical recall of emotions. Cognitive, Affective, \& Behavioral Neuroscience, 7(3), 243-250. https://doi.org/10.3758/CABN.7.3.243

Mayer, J. D., \& Gaschke, Y. N. (1988). The experience and meta-experience of mood. Journal of Personality and Social Psychology, 55(1), 102-111. https://doi.org/10.1037/00223514.55.1.102

McNair, D., Lorr, M., \& Droppleman, L. (1992). Profile of mood states (3rd ed.). San Diego: Educational \& Industrial Testing Service.

NASA. (1986). Nasa Task Load Index (TLX) v. 1.0 Manual.

Nesbit, S. M., Conger, J. C., \& Conger, A. J. (2007). A quantitative review of the relationship between anger and aggressive driving. Aggression and Violent Behavior, 12(2), 156176. https://doi.org/10.1016/j.avb.2006.09.003

Niedenthal, P. M., Krauth-Gruber, S., \& Ric, F. (2006). Psychology of emotion: interpersonal, experiential, and cognitive approaches. New York: Psychology Press.

Nikolla, D., Edgar, G., Catherwood, D., \& Matthews, T. (2018). Can bottom-up processes of attention be a source of 'interference' in situations where top-down control of attention is crucial? British Journal of Psychology, 109(1), 85-98. https://doi.org/10.1111/bjop.12251

Okutucu, S., Civelekler, M., Aparci, M., Sabanoglu, C., Dikmetas, O., Aksoy, H., ... Oto, A. (2016). Computerized dynamic pupillometry indices mirrors the heart rate variability parameters. European Review for Medical and Pharmacological Sciences, 20(10), 2099-2105. 
Pahlm, O., \& Sörnmo, L. (1984). Software QRS detection in ambulatory monitoring — a review. Medical \& Biological Engineering \& Computing, 22(4), 289-297. https://doi.org/10.1007/BF02442095

Pêcher, C., Lemercier, C., \& Cellier, J.-M. (2011). The Influence of Emotions on Driving Behavior.

Porges, S. W., Heilman, K. J., Bazhenova, O. V., Bal, E., Doussard-Roosevelt, J. A., \& Koledin, M. (2007). Does motor activity during psychophysiological paradigms confound the quantification and interpretation of heart rate and heart rate variability measures in young children? Developmental Psychobiology, 49(5), 485-494. https://doi.org/10.1002/dev.20228

Posner, M. I., \& Petersen, S. E. (1990). The Attention System of the Human Brain. Annual Review of $\quad$ Neuroscience, $\quad$ 13(1), 25-42. https://doi.org/10.1146/annurev.ne.13.030190.000325

Regan, M. A., Hallett, C., \& Gordon, C. P. (2011). Driver distraction and driver inattention: Definition, relationship and taxonomy. Accident Analysis \& Prevention, 43(5), 17711781. https://doi.org/10.1016/j.aap.2011.04.008

Riganello, F., Cortese, M. D., Arcuri, F., Quintieri, M., \& Dolce, G. (2015). How Can Music Influence the Autonomic Nervous System Response in Patients with Severe Disorder of Consciousness? Frontiers in $\quad$ Neuroscience, https://doi.org/10.3389/fnins.2015.00461

Rogé, J., Douissembekov, E., \& Vienne, F. (2012). Low Conspicuity of Motorcycles for Car Drivers: Dominant Role of Bottom-Up Control of Visual Attention or Deficit of TopDown Control? Human Factors, 54(1), 14-25. https://doi.org/10.1177/0018720811427033 
Rogé, J., El Zufari, V., Vienne, F., \& Ndiaye, D. (2015). Safety messages and visibility of vulnerable road users for drivers. Safety Science, 79, 29-38. https://doi.org/10.1016/j.ssci.2015.05.002

Rogé, J., Ndiaye, D., Aillerie, I., Aillerie, S., Navarro, J., \& Vienne, F. (2017). Mechanisms underlying cognitive conspicuity in the detection of cyclists by car drivers. Accident Analysis \& Prevention, 104, 88-95. https://doi.org/10.1016/j.aap.2017.04.006

Ruscio, D., Bos, A. J., \& Ciceri, M. R. (2017). Distraction or cognitive overload? Using modulations of the autonomic nervous system to discriminate the possible negative effects of advanced assistance system. Accident Analysis \& Prevention, 103, 105-111. https://doi.org/10.1016/j.aap.2017.03.023

Russell, J. A., Weiss, A., \& Mendelsohn, G. A. (1989). Affect Grid: A single-item scale of pleasure and arousal. Journal of Personality and Social Psychology, 57(3), 493-502. https://doi.org/10.1037/0022-3514.57.3.493

Salvia, E. (2012, December). Impact of emotional load on neurophysiologic activity and decision-making processes : application to driving (Theses). Université Claude Bernard - Lyon I.

Sarlo, M., Palomba, D., Buodo, G., Minghetti, R., \& Stegagno, L. (2005). Blood pressure changes highlight gender differences in emotional reactivity to arousing pictures. Biological Psychology, $70(3)$, 188-196. https://doi.org/10.1016/j.biopsycho.2005.01.005

Schaefer, A., Nils, F., Sanchez, X., \& Philippot, P. (2010). Assessing the effectiveness of a large database of emotion-eliciting films: A new tool for emotion researchers. Cognition \& Emotion, 24(7), 1153-1172. https://doi.org/10.1080/02699930903274322

Scherer, K. R. (2005). What are emotions? And how can they be measured? Social Science Information, 44(4), 695-729. https://doi.org/10.1177/0539018405058216 
Sirois, S., \& Brisson, J. (2014). Pupillometry: Pupillometry. Wiley Interdisciplinary Reviews: Cognitive Science, 5(6), 679-692. https://doi.org/10.1002/wcs.1323

Smallwood, J., \& Schooler, J. W. (2015). The Science of Mind Wandering: Empirically Navigating the Stream of Consciousness. Annual Review of Psychology, 66(1), 487518. https://doi.org/10.1146/annurev-psych-010814-015331

Stephens, A. N., Trawley, S. L., Madigan, R., \& Groeger, J. A. (2013). Drivers Display AngerCongruent Attention to Potential Traffic Hazards: Anger and visual attention. Applied Cognitive Psychology, 27(2), 178-189. https://doi.org/10.1002/acp.2894

Tarvainen, M. (2014). Kubios HRV user's guide version 2.2.

Task Force of The European Society of Cardiology and The North American Society of Pacing and Electrophysiology. (1996). Heart rate variability: standards of measurement, physiological interpretation and clinical use. Circulation, 93(5), 1043-1065.

Techer, F., Jallais, C., Fort, A., \& Corson, Y. (2015). Assessing the impact of anger state on the three Attentional Networks with the ANT-I. Emotion, 15(3), 276-280. https://doi.org/10.1037/emo0000028

The French Road Safety Observatory. (2016). La sécurité routière en France - Bilan de l'accidentalité de l'année 2015 [rapport public].

Valenza, G., Allegrini, P., Lanatà, A., \& Scilingo, E. P. (2012). Dominant Lyapunov exponent and approximate entropy in heart rate variability during emotional visual elicitation. Frontiers in Neuroengineering, 5. https://doi.org/10.3389/fneng.2012.00003

Valverde, L., Lera, E. de, \& Fernàndez, C. (2010). Inferencing Emotions through the Triangulation of Pupil Size Data, Facial Heuristics and Self-Assessment Techniques (pp. 147-150). IEEE. https://doi.org/10.1109/eLmL.2010.37

Wickens, C. D. (2002). Multiple resources and performance prediction. Theoretical Issues in Ergonomics Science, 3(2), 159-177. https://doi.org/10.1080/14639220210123806 
792 Wulf, G., Hancock, P. A., \& Rahimi, M. (1989). Motorcycle conspicuity: An evaluation and synthesis of influential factors. Journal of Safety Research, 20(4), 153-176.

794 https://doi.org/10.1016/0022-4375(89)90025-X

795 